\title{
Tobie Nathan: les racines, la migration et l'ethnopsychiatrie
}

Jean Martin

jean-martin[at]saez.ch
Emigré avec beaucoup de membres de la communauté juive du Caire suite à l'affaire de Suez de 1956, Tobie Nathan, après avoir passé avec sa famille par Rome, est arrivé en France en 1958, à l'âge de dix ans. Il a vécu dans la banlieue parisienne, dans une ambiance communautaire israélite. Il a intensément vécu mai 1968 et les années qui ont suivi: d'extrême gauche comme tout le monde (chez les intellectuels) à l'époque, passionné par les sciences humaines, très preneur de la libération sexuelle. Il se forme assidûment en psychanalyse (entrera en 1978 à l'Institut français de psychanalyse), ses intérêts l'orientent vers l'ethnopsychiatrie. Il crée en 1979 la première consultation de cette discipline en France, dans le service de psychiatrie de l'enfant et de l'adolescent de l'Hôpital Avicenne, alors dirigé par Serge Lebovici consultation dont les principes ont été adoptés par de nombreux centres à l'étranger.
Tobie Nathan; comment elle surgit et resurgit dans le récit de sa vie d'étudiant (au long de plusieurs étapes), de professionnel et chercheur, d'enseignant, d'homme, de père d'un fils, Michaël. Ainsi, il évoque souvent le grand-père de son grand-père, grand rabbin d'Egypte dont il porte le nom et auquel il se sent relié.

«Les humains naissent attachés. Ils ressemblent certes aux animaux, qui peuvent se mouvoir. Mais tout autant aux arbres, dont les racines les tiennent et les relient au passé le plus reculé et aux autres arbres avec lesquels ils partagent la terre.» Insistance donc sur les racines, qui pourrait surprendre à notre époque fluide, de sociétés si mobiles (en particulier dans les pays du Nord mais aussi en Asie). Il a évidemment raison pourtant: ne pas oublier les rôles que «d'où je suis issu» jouent pour chacun (ou le flou voire l'impensable de cette origine - voir plus bas ce

\section{«Le bouleversement de l'ordre familial est à la fois le plus grand bienfait et la plus grande souffrance de l'émigration.» (T. Nathan)}

Il vient de publier une autobiographie [1]. Résumé d'un observateur: «Gitan de la science, métèque, juif aimant Dieu mais aussi ses divinités connexes, Tobie Nathan traduit les mythes bibliques et les fait dialoguer avec les contes orientaux ou africains [...] Il est mû par une intuition fondamentale: la pensée est une production collective [...] Son intégration à la société française n'a jamais signifié renoncer à la mémoire du passé ni renier les cultures dont sa famille hérite: judéité, arabité, francité, africanité.» [2]

\section{Racines}

Tobie Nathan est issu d'une longue lignée rabbinique qui a vécu durant des siècles en Egypte. Egypte où, dit-il, il n'y a plus un seul Juif. Vraiment? Dans tous les cas, sa remarque fait écho à ma préoccupation, avec beaucoup d'autres devant le fait que, de manière plus ou moins brutale voire meurtrière, on voit dans plusieurs pays du Proche-Orient une sorte de purification ethnique: cette région du monde perd rapidement sa dimension historique de multiculturalité, notamment au plan religieux.

J'ai été frappé de voir combien son appartenance originelle prend de place dans l'autobiographie de qu'il dit de Viviane). Après une rencontre avec un guérisseur du Bénin, il observe: «Apprendre, c'est s'imprégner des objets, des substances, des paroles, des lieux qui nous ont vu naître.»

Pourtant, bien sûr, les racines sont bousculées. Un exemple: évoquant l'exode rural et l'évolution socio-politique de populations françaises auparavant très catholiques, dans les années 1960: «Le parti communiste fournissait au peuple, ces immigrés de

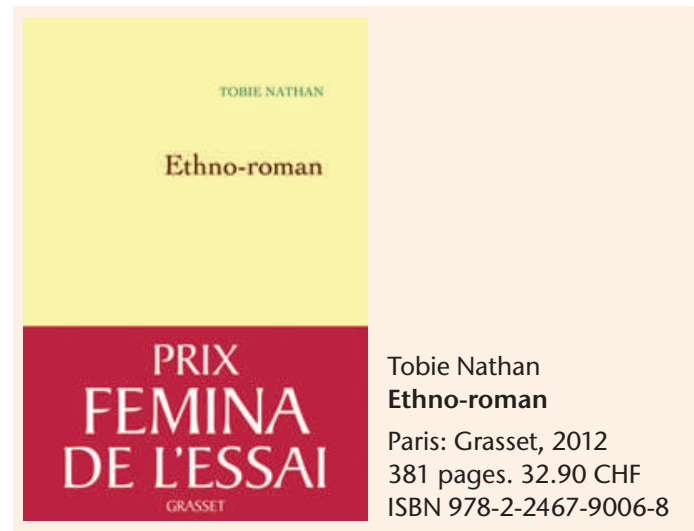




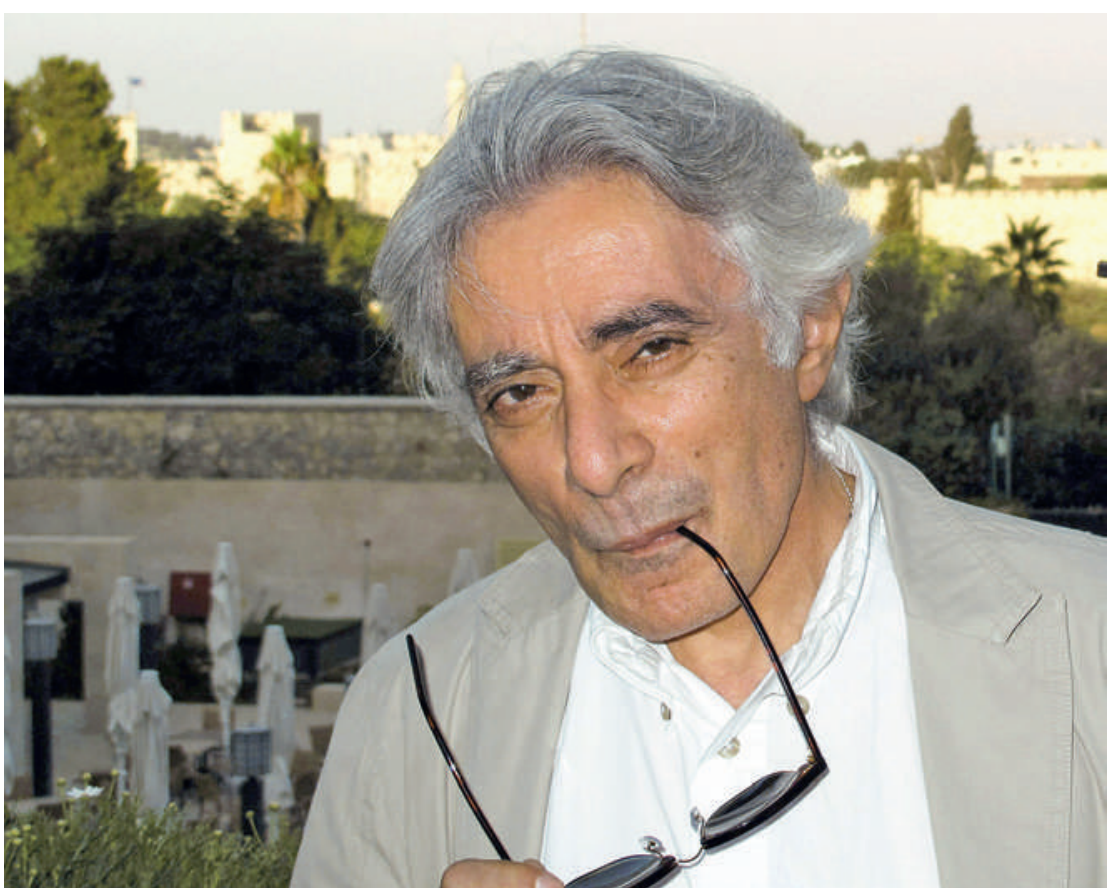

Tobie Nathan, un pied de chaque côté de la Méditerranée et l'esprit fouillant une multiplicité de cultures. taires que l'enfant a absorbées sans effort. L'enfant est un père pour le père qui lui demande son chemin ou la traduction d'un mot.» «Nos parents n'avaient aucune idée de la hiérarchie des études, des manières d'entrer dans telle profession. C'était nous qui leur expliquions le monde où ils vivaient. Nous n'étions pas pour autant des êtres sans noms et sans familles. Notre héritage était fait de traditions et de mythes, mais ces richesses étaient désactivées.» «Tel est si souvent le destin des enfants dans les familles migrantes, guides de leurs propres parents dont l'âme parasitée est hypnotisée par ce monde trop nouveau.»

Grande bousculade, tohu-bohu des connaissances et des valeurs: «Le bouleversement de l'ordre familial est à la fois le plus grand bienfait et la plus grande souffrance de l'émigration.» «Fascination de l'émigré pour cette vertigineuse liberté qui s'ouvre soudain devant lui - liberté de penser, de faire, d'enfreindre... En contrepartie le monde devient désordre.»

\section{Psychanalyse et psychothérapie - Soigner}

Tobie Nathan a suivi le séminaire de Georges Devereux, «un découvreur, un décapsuleur, un maniaque de l'eurêka»: grand esprit protéiforme d'origine hongroise, qui a vécu plusieurs années aux Etats-Unis où il pratiquait la psychanalyse, avant de revenir en France et d'y fonder l'ethnopsychiatrie. Après un compagnonnage étroit durant une dizaine d'années, il y a eu rupture brutale, dont Nathan estime qu'elle était la manière pour Devereux - ainsi qu'il l'avait dit longtemps auparavant - de l'instituer comme son successeur: «'ai considéré qu'il s'agissait de son dernier enseignement, m'apprendre à partir.»

Il discute les enseignements de son activité de thérapeute. «Le métier d'homme n'est rien d'autre que la tentative toujours répétée de percevoir ses propres singularités et de les apprivoiser. Je mettrai longtemps à le comprendre.» «Je pressentais que les pathologies des patients s'améliorent à l'aune des sacrifices consentis par les thérapeutes [...] La seule force qui soigne les patients en psychothérapie, c'est la furor sanandi de leur thérapeute, sa passion de guérir. Mais il est une seconde condition, tout aussi active: les thérapeutes se révèlent efficaces lorsque les cures qu'ils conduisent leur permettent de connaître le monde. Sitôt qu'ils n'apprennent plus, sitôt qu'ils savent, leurs capacités thérapeutiques déclinent.» Pas sûr que cette remarque sur la furor sanandi fasse l'unanimité (je me souviens d'un confrère très impliqué dans la prise en charge des toxicomanes disant qu'il convenait aussi de s'en méfier...).

Racontant une rencontre avec un guérisseur du Proche-Orient dont la femme est mentalement dérangée et qui malgré beaucoup d'efforts n'est jamais parvenu à la soigner: «On ne peut soigner sa propre famille. Un couteau pourrait-il se couper lui-même?»,

disait-il. Serait-ce pour une raison similaire que chez

\begin{abstract}
père qui ne parvient pas à intégrer les règles élémen-
Migration

'humanité. Sous des formes multiples. Elles repr compris aujourd'hui [3]. En passant, je mentionn une intéressante monographie, publiée à l'occasion des 125 ans de la Policlinique universitaire de Laucanton de Vaud depuis le XIX ${ }^{\mathrm{e}}$ siècle [4].

Tobie Nathan jette un regard sur sa propre expérience migratoire (comme le fait Jean-Claude Métraux - [3]), relevant notamment ce que les profesconnaissent bien: la parentification des enfants visà-vis de leurs géniteurs insuffisamment intégrés.

«Le père est un enfant aux yeux de son fils - ce
\end{abstract} père qui ne parvient pas à integrer les règles élémen- 
nous aussi on recommande de ne pas soigner ses proches?!

\section{Enseignement}

Au long du livre, l'auteur rapporte ses expériences d'enseignant. Touchant des thèmes souvent discutés dans les institutions de formation, Facultés de médecine et autres, et à propos desquels il n'y a pas une seule bonne réponse (comme dans tous les domaines de la vie!).

\section{Ethnopsychiatrie}

A propos d'une guérison survenue au Brésil dans un rite candomblé: «Sa guérison lui avait indiqué qu'il avait été malade de s'être pensé homme nu, n'appartenant qu'à lui seul, né de sa propre volonté.» Les psychiatres systémiciens et tous les soignants qui donnent attention au milieu du patient, dans un sens large, pourront reprendre à leur compte.

Pour terminer, une citation qui donne une idée de l'ambiance de l'ouvrage et des questions interpel-

\section{«C'était un tour de force que de parvenir à intéresser des étudiants obsédés par leurs examens de biologie et d'anatomie à ces questions qui mêlaient philosophie et pratique quotidienne.»}

«C'est au séminaire de Georges Devereux que j'ai acquis la conviction que pour former les étudiants il n'est besoin d'aucun programme, d'aucun plan seulement de professeurs, de ceux qu'on appelait autrefois des maîtres.» Oui et non... C'est une vraie chance d'avoir connu des maîtres charismatiques et d'être marqués par eux, mais on ne peut guère nier l'utilité de programmes structurés.

Une regrettable observation souvent faite, à laquelle l'accent actuel sur les Medical humanities espère répondre: «J'avais commencé à enseigner la psychologie médicale à la Faculté de médecine de Bobigny. C'était un tour de force que de parvenir à intéresser des étudiants obsédés par leurs examens de biologie et d'anatomie à ces questions qui mêlaient philosophie et pratique quotidienne.»

A propos d'hypnose, qu'il a apprise de Léon Chertok qui en a été un praticien reconnu: «Lorsqu'on lui posait des questions, il répondait qu'il y avait un mystère dans l'hypnose qui ne tenait ni à la suggestion ni au transfert; en matière d'hypnose il fallait accepter de ne pas savoir.» Pourquoi pas, bien que la généralisation de telle acceptation soit difficilement envisageable. lantes qu'il pose: «Chaque dieu créateur a été à l'origine d'un type de division; il se reconnaît à sa façon de fracturer le noyau compact des origines. Les dieux ont créé les hommes et, à parcourir le monde, il est facile de constater que des dieux différents ont créé des hommes différents.» Puis, à propos d'une patiente descendante d'esclaves: «Durant cette consultation j'avais expédié Viviane aux temps impensables où l'une de ses ancêtres, une esclave, avait été assaillie par l'homme blanc... Les hommes se mélangent de gré ou de force mais leurs dieux restent trempés du même métal, imposent toujours les mêmes contraintes à leurs fidèles. Ce sont les dieux qu'il nous faut désormais éduquer, à qui il devient indispensable d'enseigner la multiplicité.»

\section{Références}

1 Nathan T. Ethno-roman. Paris: Grasset; 2012.

2 Benkirane R. Article dans Le Temps (Genève), 10 novembre 2012, p. 42.

3 Métraux JC. La migration comme métaphore. Paris: Ed. La Dispute; 2011.

4 Garibian T, Barras V. Migration et système de santé vaudois, du $19^{\text {e }}$ siècle à nos jours. Lausanne: Editions BHMS (Institut univ. d'histoire de la médecine et de la santé publique); 2012. 